Variable $1 \quad$ User ID

Name (GUI ENG) User ID

Name (database) UserID

UMLS ID

C1708731

Type

String

Length max char 25

Unit

Group

User_db

Data entry

Autogenerate

Comments

Terminology

Index

Codes:

Category1

Category2

Category3

Category4

Category5

Category6

Category7

Category8

Category9

Category10 
Variable 2

\section{User Role}

Name (GUI ENG) User Role

Name (database) UserRole

UMLS ID

C1883484

Type

String

Length max char 50

Unit

Group

User_db

Data entry

Dropdown

Comments

Terminology

Index

User_categories

Codes:

Category1

Health care professional

Category2

Burn injury expert

Category3

Administrator

Category4

Category5

Category6

Category7

Category8

Category9

Category10 
Variable 3

First Name

Name (GUI ENG) First Name

Name (database) FirstName

UMLS ID

C1443235

Type

String

Length max char 25

Unit

Group

Data entry

User_db

Comments

Terminology

Index

NA

Codes:

Category1

Category2

Category3

Category4

Category5

Category6

Category7

Category8

Category9

Category10 
Variable 4

\section{Last Name}

Name (GUI ENG) Last Name

Name (database) LastName

UMLS ID

C1301584

Type

String

Length max char 50

Unit

Group

Data entry

User_db

Comments

Terminology

Index

NA

Codes:

Category1

Category2

Category3

Category4

Category5

Category6

Category7

Category8

Category9

Category10 
Variable $5 \quad$ Email address

Name (GUI ENG) Email address

Name (database) EmailAddress

UMLS ID

C1705961

Type

String

Length max char

Unit

Group

Data entry

User_db

Comments

Terminology

Index

NA

Codes:

Category1

Category2

Category3

Category4

Category5

Category6

Category7

Category8

Category9

Category10 
Variable 6

Mobile phone number

Name (GUI ENG) Mobile phone number

Name (database) MobilePhoneNumber

UMLS ID

C1549617

Type

Numeric

Length max char 50

Unit

Group

Data entry

User_db

Comments

Terminology

Index

NA

Category1

Category2

Category3

Category4

Category5

Category6

Category7

Category8

Category9

Category10 
Variable 7

\section{Address}

Name (GUI ENG) Address

Name (database) Address

UMLS ID

C1442065

Type

String

Length max char 100

Unit

Group

User_db

Data entry

Textbox

Comments

Terminology

Index

NA

Category1

Category2

Category3

Category4

Category5

Category6

Category7

Category8

Category9

Category10 
Variable 8

\section{Patient ID}

Name (GUI ENG) Patient ID

Name (database) PatientID

UMLS ID

C2706990

Type

String

Length max char

Unit

Group

Data entry

Patient_db

Comments

Terminology

Index

NA

Category1

Category2

Category3

Category4

Category5

Category6

Category7

Category8

Category9

Category10 
Variable $9 \quad$ Patient age

Name (GUI ENG) Patient age

Name (database) PatientAge

UMLS ID C3166952

Type Numeric

Length max char 25

Unit In months if age $<1$ year and in years if age $>=1$ year

Group Patient_db

Data entry Textbox

Comments

Terminology

Index

Patient_age

Category1

Integer

Category2

Unknown

Category3

Category4

Category5

Category6

Category7

Category 8

Category9

Category10 
Variable $10 \quad$ Patient gender

Name (GUI ENG) Patient gender

Name (database) PatientGender

UMLS ID C0079399

Type String

Length max char 25

Unit

Group

Data entry

Patient_db

Comments

Dropdown

Terminology

Index

PatientGender

Category1

Female

Category2

Male

Category3

Unknown

Category4

Category5

Category6

Category7

Category8

Category9

Category10 
Variable $11 \quad$ Patient weight

Name (GUI ENG) Patient weight

Name (database) PatientWeight

UMLS ID C0005910

Type Numeric

Length max char 25

Unit

In kilograms

Group

Patient_db

Data entry

Dropdown

Comments

Terminology

Index

Patient_weight

Category1

Integer

Category2

Unknown

Category3

Category4

Category5

Category6

Category7

Category8

Category9

Category10 
Variable $12 \quad$ Time since injury

Name (GUI ENG) Time since injury

Name (database) TimeSincelnjury

UMLS ID

C0449246

Type

String

Length max char

Unit

Group

Data entry

Patient_db

Comments

Terminology

Index

TimeSincelnjury

Category1

1-24 hours ago

Category2

24-72 hours ago

Category3

72 hrs-1 week ago

Category4 >than 1 week ago

Category5

Unknown

Category6

Category7

Category8

Category9

Category10 


\section{Variable $13 \quad$ Cause of injury}

$\begin{array}{ll}\text { Name (GUI ENG) } & \text { Cause of injury } \\ \text { Name (database) } & \text { CauseOfInjury } \\ \text { UMLS ID } & \text { C2025548 } \\ \text { Type } & \text { String } \\ \text { Length max char } & \\ \text { Unit } & \\ \text { Group } & \text { Patient_db } \\ \text { Data entry } & \text { Dropdown } \\ \text { Comments } & \\ \text { Terminology } & \text { ICD10 } \\ \text { Index } & \text { Cause_of_injury } \\ \text { Category1 } & \text { Fire/flame } \\ \text { Category2 } & \text { Hot object } \\ \text { Category3 } & \text { Hot water } \\ \text { Category4 } & \text { Chemical } \\ \text { Category5 } & \text { Frozen object } \\ \text { Category6 } & \text { Electricity } \\ \text { Category7 } & \text { Other } \\ \text { Category8 } & \text { Unknown } \\ \text { Category9 } & \\ \text { Category10 } & \end{array}$


Variable $14 \quad$ Total burn surface area

Name (GUI ENG) Total burn surface area

Name (database) TBSA

UMLS ID

C0433297

Type

String

Length max char

Unit

Group

Data entry

Patient_db

Comments

Terminology

Index

Total_burn_surface_area

Category1

Category2

Category3

Category4

Category5

Category6

Category7

Category8

Category9

Category10 
Variable $15 \quad$ Body part

Name (GUI ENG) Body part

Name (database) BodyPart

UMLS ID

C0229962

Type

String

Length max char

Unit

Group

Data entry

Patient_db

Comments

Terminology

Index

Body_parts

Category1

Head

Category2

Chest

Category3

Upper arm Across joint

Category4

Upper arm No across joint

Category5

Lower arm Across joint

Category6

Lower arm Not across joint

Category7

Hand Across joint

Category8

Hand Not across joint

Category9

Abdomen

Category10

Genitals

Category11

Upper leg Across joint

Category12

Upper leg Not across joint

Category13

Lower leg Across joint

Category14

Lower leg Not across joint

Category15

Foot 
Variable $16 \quad$ Side

Name (GUI ENG) Side

Name (database) Side

UMLS ID

C0441987

Type

String

Length max char

Unit

Group

Data entry

Patient_db

Comments

Terminology

Index

Side

Category1

Right

Category2

Left

Category3

Anterior (front)

Category4

Posterior (back)

Category5

Category6

Category7

Category8

Category9

Category10 
Variable 17

Pre-Existing Condition

Name (GUI ENG) Pre-Existing Condition

Name (database) PreExistingCondition

UMLS ID

C0521987

Type

String

Length max char

Unit

Group

Data entry

Patient_db

Comments

Terminology

Index

Pre-Existing Condition

Category1

Diabetes

Category2

HIV

Category3

Tuberculosis

Category4

Ischaemic heart disease

Category5

Cancer

Category6

Nuntrintional deficiency

Category 7

No/poor immunization history

Category8

Other

Category9

Category10 
Variable 18

\section{Additional comments}

Name (GUI ENG) Additional comments

Name (database) AdditionalComments

UMLS ID

C1830770

Type

String

Length max char

Unit

Group

Patient_db

Data entry

Textbox

\section{Comments}

Terminology

Index

Category1

Category2

Category3

Category4

Category5

Category6

Category7

Category8

Category9

Category10 
Variable $19 \quad$ Where to treat

Name (GUI ENG) Where to treat

Name (database) WhereToTreat

UMLS ID

C2585021

Type

String

Length max char

Unit

Group

Data entry

Management_db

Comments

Options

Terminology

Index

Where_to_treat

Category1

Discharge

Category2

Review in own facility

Category3

Admit own facility

Category4

Refer local referral hospital

Category5

Refer burn unit

Category6

Category7

Category8

Category9

Category10 
Variable 20

\section{Fluid Therapy}

Name (GUI ENG) Fluid Therapy

Name (database) FluidTherapy

UMLS ID

C0016286

Type

String

Length max char

Unit

Group

Data entry

Management_db

Comments

Terminology

Index

Fluids

Category1

Glucose (basic needs)

Category2

Colloids

Category3

Ringerlactate

Category 4

Blood

Category5

Category6

Category7

Category8

Category9

Category10 
Variable $21 \quad$ Volume of fluid

Name (GUI ENG) Volume of fluid

Name (database) VolumeOfFluid

UMLS ID C0449971

Type Numeric

Length max char Unit $\mathrm{ml}$

Group Management_db

Data entry Textbox

\section{Comments}

Terminology

Index

Category1

Category2

Category3

Category4

Category5

Category6

Category7

Category8

Category9

Category10 
Variable $22 \quad$ Drugs

Name (GUI ENG) Drugs

Name (database) Drugs

UMLS ID

C0013227

Type

String

Length max char

Unit

Group

Data entry

Management_db

Comments

Terminology

Index

Category1

Category2

Category3

Category4

Category5

Category6

Category7

Category8

Category9

Category10 
Variable 23 Drug dose

Name (GUI ENG) Drug dose

Name (database) DrugDose

UMLS ID C0678766

Type String

Length max char

Unit

Group

Data entry

Management_db

Comments

Terminology

Index

Category1

Category2

Category3

Category4

Category5

Category6

Category7

Category8

Category9

Category10 
Variable $24 \quad$ Burn dressings

Name (GUI ENG) Burn dressings

Name (database) BurnDressings

UMLS ID

C0180518

Type

String

Length max char

Unit

Group

Data entry

Management_db

Comments

Terminology

Index

Category1

Category2

Category3

Category4

Category5

Category6

Category7

Category8

Category9

Category10 
Variable $25 \quad$ Specific instructions

Name (GUI ENG) Specific instructions

Name (database) Specificlnstructions

UMLS ID

C1442085

Type

String

Length max char

Unit

Group

Data entry

Management_db

Comments

Terminology

Index

Category1

Category2

Category3

Category4

Category5

Category6

Category7

Category8

Category9

Category10 